\section{D $\quad$ A DYSTROPHIN-DEPENDENT LOSS OF NEURONAL NITRIC OXIDE SYNTHASE IN ATRIAL FIBRILLATION (AF) PROMOTES ELECTRICALREMODELLING AND CREATES A SUBSTRATE FOR THE MAINTENANCE OF AF}

S N Reilly, X Liu, R Carnicer, R Jayaram, N C Surdo, C Ratnatunga, R Sayeed, R De Silva, S Verheule, U Schotten, B Casadei The University of Leeds, United Kingdom

doi:10.1136/heartjnl-2013-304019.277

Rationale Nitric oxide production in the heart is involved in the regulation of ion channels activity, myocardial perfusion and thrombogenesis. Atrial fibrillation (AF) has been associated with reduced myocardial nitric oxide (NO) bioavailability; however, the mechanisms and the consequences of this phenomenon remain to be elucidated.

Methods and Results We evaluated the activity, protein content, and localization of constitutive $\mathrm{NO}$ synthases (NOS) in atrial tissue from 62 patients with permanent AF and 148 controls in normal sinus rhythm (SR), and in goats after 2 weeks (2W-AF, $n=15)$ or 6 months (6M-AF, $n=10)$ of pacing-induced AF (vs. sham surgery \& $\mathrm{SR}, \mathrm{n}=14)$. NOS activity was uncoupled in right atrial (RA) tissue from AF patients and 6M-AF goats, both of which also exhibited an ipsilateral reduction in the NOS cofactor tetrahydrobiopterin (BH4) and an increase in arginase activity (HPLC). Although ex vivo $\mathrm{BH} 4$ and arginine supplementation reversed NOS uncoupling, atrial NOS activity remained profoundly suppressed in AF due to a progressive decrease in the "neuronal" isoform of NOS (nNOS) in atrial cardiomyocytes (by $65 \%$ in $2 \mathrm{~W}-\mathrm{AF}, 86 \%$ in $6 \mathrm{M}-\mathrm{AF}$ goats and $62 \%$ in patients with $\mathrm{AF}$ ). Atrial nNOS mRNA expression (qRT-PCR) did not differ between AF and SR; however, nNOS ubiquitination was increased in AF. Atrial nNOS protein content was partially restored by inhibition of proteasomal activity with MG132 but not by inhibiting the autophagy-lysosomal pathway with bafilomycin A1 or by pre-treatment with BH4. nNOS signalling and protein stability in the cardiac muscle depends on the enzyme's anchoring to the dystrophin-syntrophin complex at the sarcolemmal membrane. In the fibrillating atrial myocardium we observed a significant reduction in dystrophin (by $56 \%$ ) and its associated proteins, $1 \alpha$ syntrophin and caveolin-3; by contrast no difference in the dystrophin complex was observed in the atrial myocardium of $\mathrm{nNOS}^{-/-}$mice. Immunoprecipitation showed co-localisation of $\mathrm{nNOS}$ with dystrophin in SR, but not in $\mathrm{AF}$.

To assess whether loss of $\mathrm{nNOS}$ and reduced $\mathrm{NO}$ bioavailability played a role in the AF-induced atrial electrical remodelling; we evaluated the effect of nNOS inhibition or gene deletion in human and murine atrial myocytes. In RA myocytes from patients in SR (but not in patients with AF), nNOS inhibition shortened the action potential duration (APD) by $40 \%$, and decreased the rate dependent-adaptation of APD (both of which are a hallmark of AF-induced electrical remodelling). These findings were recapitulated in murine right atrial myocytes in the presence of nNOS gene deletion or inhibition. In mice, nNOS gene deletion was sufficient to induce a 2 -fold increase in the likelihood of developing AF in response to electrical stimulation.

Conclusions - These data indicate that dystrophin-dependent loss of myocardial $\mathrm{nNOS}$ in $\mathrm{AF}$ is sufficient to produce an atrial electrical substrate that increases both inducibility and maintenance of this arrhythmia. Therapeutic strategies aimed at preserving or restoring the dystrophin complex may have a profound effect on $\mathrm{NO}$ bioavailability and the electrical substrate of AF. 